\title{
BMJ Global Health Effects of food policy actions on Indigenous Peoples' nutrition-related outcomes: a systematic review
}

\author{
Jennifer Browne, ${ }^{1}$ Mark Lock, ${ }^{1}$ Troy Walker, ${ }^{1}$ Mikaela Egan, ${ }^{2}$ Kathryn Backholer ${ }^{1}$
}

To cite: Browne J, Lock M, Walker T, et al. Effects of food policy actions on Indigenous Peoples' nutrition-related outcomes: a systematic review. BMJ Global Health 2020;5:e002442. doi:10.1136/ bmjgh-2020-002442

Handling editor Stephanie M Topp

- Additional material is published online only. To view please visit the journal online (http://dx.doi.org/10.1136/ bmjgh-2020-002442).

Received 1 March 2020 Revised 12 June 2020 Accepted 18 June 2020
Check for updates

(C) Author(s) (or their employer(s)) 2020. Re-use permitted under CC BY-NC. No commercial re-use. See rights and permissions. Published by BMJ.

${ }^{1}$ Deakin University, Global Obesity Centre, Institute for Health Transformation, Geelong, Victoria, Australia

${ }^{2}$ Victorian Aboriginal Community Controlled Health Organisation, Melbourne, Victoria, Australia

Correspondence to

Dr Jennifer Browne;

jennifer.browne@deakin.edu.au

\section{ABSTRACT}

Introduction Indigenous Peoples worldwide endure unacceptable health disparities with undernutrition and food insecurity often coexisting with obesity and chronic diseases. Policy-level actions are required to eliminate malnutrition in all its forms. However, there has been no systematic synthesis of the evidence of effectiveness of food and nutrition policies for Indigenous Peoples around the world. This review fills that gap.

Methods Eight databases were searched for peerreviewed literature, published between 2000 and 2019. Relevant websites were searched for grey literature. Articles were included if they were original studies, published in English and included data from Indigenous Peoples from Western colonised countries, evaluated a food or nutrition policy (or intervention), and provided quantitative impact/outcome data. Study screening, data extraction and quality assessment were undertaken independently by two authors, at least one of whom was Indigenous. A narrative synthesis was undertaken with studies grouped according to the NOURISHING food policy framework.

Results We identified 78 studies from Canada, Australia, Aotearoa/New Zealand and the USA. Most studies evaluated targeted interventions, focused on rural or remote Indigenous communities. The most effective interventions combined educational strategies with policies targeting food price, composition and/or availability, particularly in retail and school environments. Interventions to reduce exposure to unhealthy food advertising was the only area of the NOURISHING framework not represented in the literature. Few studies examined the impact of universal food policies on Indigenous Peoples' diets, health or well-being.

Conclusion Both targeted and universal policy action can be effective for Indigenous Peoples. Actions that modify the structures and systems governing food supply through improved availability, access and affordability of healthy foods should be prioritised. More high-quality evidence on the impact of universal food and nutrition policy actions for Indigenous Peoples is required, particularly in urban areas and in the area of food marketing.

\section{INTRODUCTION}

The United Nations Declaration on the Rights of Indigenous Peoples affirms their right to the highest attainable standard of

\section{Key questions}

What is already known?

- Indigenous Peoples worldwide experience unacceptable health disparities. To improve food and nutrition-related health outcomes for Indigenous Peoples, both targeted (Indigenous-specific) and universal (population-wide) policy action is required.

\section{What are the new findings?}

- This is the first international review of food and nutrition interventions among colonised Indigenous Peoples in Western nations. The most effective interventions combined strategies to promote food affordability, healthy food and beverage provision, healthy food retail systems and environments; and nutrition knowledge and skills. Targeted approaches were effective when community directed, participatory, multifaceted and culturally relevant. Universal approaches were effective when they addressed the structures and systems in which people access food.

\section{What do the new findings imply?}

- Targeted approaches should combine strategies to improve food affordability, healthy food environments and nutrition knowledge and skills. The evidence for universal approaches is limited but promising approaches include:

- Mandatory rather than voluntary food reformulation targets.

- Interpretive food labelling systems applied across all products.

- Food pricing policies that both incentivise healthy products and disincentivise unhealthy foods and beverages.

- Robust intervention monitoring, evaluation and statistical analysis is required to determine the differential impact of population-wide policy actions on Indigenous Peoples, including in urban areas.

health. ${ }^{1}$ However, many Indigenous Peoples globally continue to experience unacceptable health and social inequities compared with their non-Indigenous counterparts. ${ }^{2}$ In highincome countries such as Canada, Australia, Aotearoa (New Zealand) and the USA, life expectancy at birth for Indigenous Peoples 
is 5-10 years lower than for other citizens. ${ }^{2}$ These inequities reflect the histories of colonisation, dispossession, marginalisation and disruption of cultures and kinship systems. ${ }^{23}$ These histories interact with and are amplified by contemporary socioeconomic disparities as well as interpersonal and institutional racism. ${ }^{3}$

Indigenous Peoples' traditional food systems have protected human health and natural environments for millennia. Before their lands were colonised, Indigenous peoples worldwide maintained healthy and sustainable food systems, grounded in transgenerational knowledge of lands, waterways, seasonal food sources, sophisticated agricultural practices and food preparation methods. ${ }^{4}$ Traditional foods were derived from biodiverse plant and animal sources, high in protein, fibre and micronutrients and low in fat, sugar and salt. ${ }^{5}$ However, for many Indigenous peoples, food represents more than a source of nutrients; it is intimately connected to land, family, history and culture, as well as to social, emotional and spiritual well-being. ${ }^{5}$ Consequently, precolonial Indigenous food systems were self-determined, ecologically sustainable and provided healthy, varied diets which protected population health. ${ }^{4}$

European colonisation has severely disrupted Indigenous food systems in many countries. Dispossession of Indigenous Peoples from their homelands, introduction of industrial food production, processing and distribution has manifested in rapid Westernisation of food environments and dietary patterns. ${ }^{4-6}$ The transition from traditional to industrialised diets has increased the prevalence of obesity, type 2 diabetes and other diet-related chronic diseases globally, but especially for Indigenous populations. ${ }^{4}$ For many Indigenous peoples, food security is undermined by environmental degradation, loss of biodiversity and insufficient access to healthy food and other social and economic resources required for health, particularly in rural, remote and Arctic communities. ${ }^{5-8}$ As a result, nutrition-related health conditions disproportionately affect many Indigenous populations throughout the life course. ${ }^{2}{ }^{7-9}$ Despite these inequities, Indigenous Peoples worldwide maintain valuable ecological knowledge and continue to advocate for greater control over their food systems. ${ }^{46}$

Malnutrition, including both undernutrition and obesity, is a leading contributor to the burden of disease worldwide. ${ }^{9} 10$ Additionally, unsustainable Western food systems are increasingly recognised as a key driver of environmental degradation and climate change. ${ }^{11}$ In response, the United Nations has declared a Decade of Action on Nutrition and expert panels have called for multilevel, multisectoral policy responses to improve food environments. ${ }^{12}$ Governments around the world are increasingly implementing food and nutrition policy actions to reduce the impact of the health, environmental and economic burden caused by unhealthy diets.

A range of different food and nutrition policy options exist. These include policies targeting individual behaviour change as well as the systems and environments framing dietary norms. ${ }^{13} 14$ The NOURISHING framework outlines 10 areas for policy action: nutrition labelling; offering healthy food in specific settings; using economic tools (eg, taxes/subsidies); restricting unhealthy food marketing; improving the quality of the food supply; supply chain interventions; healthier retail environments; information campaigns; nutrition advice and counselling; and giving education/skills. ${ }^{15}$ Policies may target specific groups (usually of higher risk) or take a universal approach and target whole populations and/or settings (indiscriminate of risk), both of which are necessary to reduce inequities. Targeted policies aim to improve the health of the most disadvantaged groups. Conversely, universal policies act across sociodemographic groups and have potential to improve population health but may be markedly less effective for individuals with limited social and economic resources. ${ }^{13}$

Indigenous Peoples have the right to be actively involved in policy decisions which affect them, including the development of health priorities and strategies. ${ }^{1}$ Therefore, Indigenous Peoples should be involved in the determination of policy actions (both targeted and universal) to improve population nutrition. There is an urgent need for evidence-based, equitable public health policies. However, there have been no systematic reviews of the impact of population food and nutrition policies on Indigenous peoples around the world. In this review, we synthesised the evidence on the effectiveness of food and nutrition policy interventions on Indigenous Peoples' diets and nutrition-related health outcomes.

\section{METHODS}

This review followed the Preferred Reporting Items for Systematic Reviews and Meta-analyses Equity guidelines, ${ }^{16}$ and the protocol was registered with PROSPERO (\#CDR42019142677).

\section{Search strategy}

We searched eight electronic databases for peer-reviewed articles published between January 2000 and December 2019: MEDLINE, Embase, PsycINFO, CINAHL, Informit, Scopus, Global Health and Econlit. The International Journal of Indigenous Health, the Journal of Indigenous Wellbeing, the Indigenous Policy Journal, the First Peoples' Child \& Family Review and the New Zealand Journal of Indigenous Scholarship were hand-searched for non-indexed articles. We searched for grey literature via Google Scholar (the first 300 hits), the Australian Indigenous HealthInfoNet, the Indigenous Studies Portal, the Indigenous Knowledge Network for Infant, Child and Family Health, the NOURISHING database and national government websites from Australia, Aotearoa/New Zealand, Canada and the USA. We contacted experts in the field of Indigenous health and nutrition research from Australasia and North America for unpublished evaluation reports. Reference lists of identified papers were scanned for additional resources. 
We combined four sets of search terms, which described Indigenous populations, food policy interventions, nutrition-related outcomes and evaluation study designs. Where possible, relevant subject headings were included in each database search. Each set of search terms and subject headings was combined with the Boolean operator 'OR', then the results of each set were combined with the operator 'AND'. The MEDLINE search strategy is available in online supplementary file 1 .

\section{Study selection and inclusion criteria}

1. Population-Indigenous peoples of Western colonised countries, including studies targeting only Indigenous participants and studies of mixed populations with analyses stratified by ethnicity.

2. Intervention-study examined a policy intervention, implemented by government or an organisation (eg, school, store) aimed at improving food consumption or nutrition.

3. Outcome-quantitative impact/outcome data on behaviour, food/nutrient intake, anthropometry, biochemical indicators or diet-related health outcomes were reported.

4. Study design-evaluations of intervention impact or effectiveness. Reviews, descriptive studies, protocols, modelling and cost-effectiveness analyses were excluded.

Study screening and selection was undertaken independently by two researchers, at least one of whom was Indigenous. Articles not meeting all of these criteria were excluded. Articles unavailable in English were also excluded. Disagreements regarding study inclusion were resolved through discussion with a third researcher until consensus was reached.

\section{Data extraction and quality assessment}

A data extraction template was developed to compare study settings, interventions and evaluation results. Data were extracted from included studies, including publication details, study setting, participant characteristics, intervention type, study design, setting, duration, sample size, intervention strategies, Indigenous involvement in intervention design/implementation and relevant outcomes, including the differential impact between Indigenous and non-Indigenous participants, where applicable. The template was piloted by three reviewers with two studies each. Data extraction was completed for all included studies by two reviewers, at least one of whom was Indigenous. Results were cross-checked and discrepancies were resolved through discussion.

Quality assessment was undertaken using the relevant Joanna Briggs Institute (JBI) critical appraisal tool. ${ }^{17} \mathrm{JBI}$ has separate tools for appraising different study designs. Quality assessment was undertaken independently by two reviewers, and disagreements were resolved through discussion and/or consultation with a third researcher. Studies were classified as high quality if more than $80 \%$ of applicable appraisal characteristics were endorsed, moderate quality if $50 \%-80 \%$ of characteristics were met and low quality if less than $50 \%$ were achieved.

\section{Data synthesis}

A narrative data synthesis was undertaken due to the diverse range of interventions and study designs reviewed. The outcomes reported were heterogeneous, therefore, a meta-analysis was not possible. Instead, included studies were grouped and synthesised according to the relevant policy areas of the NOURISHING framework. Although the NOURISHING framework was not specifically designed for Indigenous contexts, it highlights different categories of population-wide food and nutrition policy action, and thus provided a useful framework for organising the findings of our review.

\section{Patient and public involvement}

This review was undertaken by a team of Indigenous and non-Indigenous health researchers and practitioners with expertise in public health, nutrition, Indigenous health and cultural safety. It was part of a larger research project designed in partnership with the Victorian Aboriginal Community Controlled Health Organisation (VACCHO). The findings of this review have been presented to VACCHO staff to inform future research, practice and advocacy.

\section{RESULTS}

The literature search identified 5276 records after duplicates were removed. After initial screening, we assessed 158 publications for eligibility. Of these, 78 studies met the inclusion criteria and were included in this review (figure 1).

These 78 studies have been summarised in online supplementary table 2 and grouped according to the policy areas of the NOURISHING framework (table 1). There were 5 articles examining food labelling, ${ }^{18-22}$ 21 relating to healthy food provision (predominantly in schools), ${ }^{23-43} 10$ reporting economic interventions (predominantly subsidies/discounts) ${ }^{44-53} \quad 10$ evaluating food composition strategies (mainly food fortification), ${ }^{54-63} 10$ food retail studies, ${ }^{64-73} 6$ food system/supply chain interventions, ${ }^{54-62} 4$ information/public awareness campaigns, ${ }^{74-77} 4$ nutrition counselling/advice evaluations ${ }^{78-81}$ and 8 education/skill development interventions. ${ }^{82-89}$ No studies evaluated the impact of unhealthy food marketing restrictions on Indigenous People. This was the only area of the NOURISHING framework not represented in this review.

A diverse range of Indigenous Peoples were described in the literature. This included Aboriginal and Torres Strait Islander Australians; First Nations, Inuit and Inuvialuit Canadians; Native American and Alaskan Native tribes; and Aotearoa Māori. Most articles were from Australia $(\mathrm{n}=31)$, followed by Canada $(n=22)$, the USA $(n=15)$ and Aotearoa $(n=10)$. Twenty-three studies included urban Indigenous People, the majority of studies $(n=55)$, however, focused on rural or remote communities. 


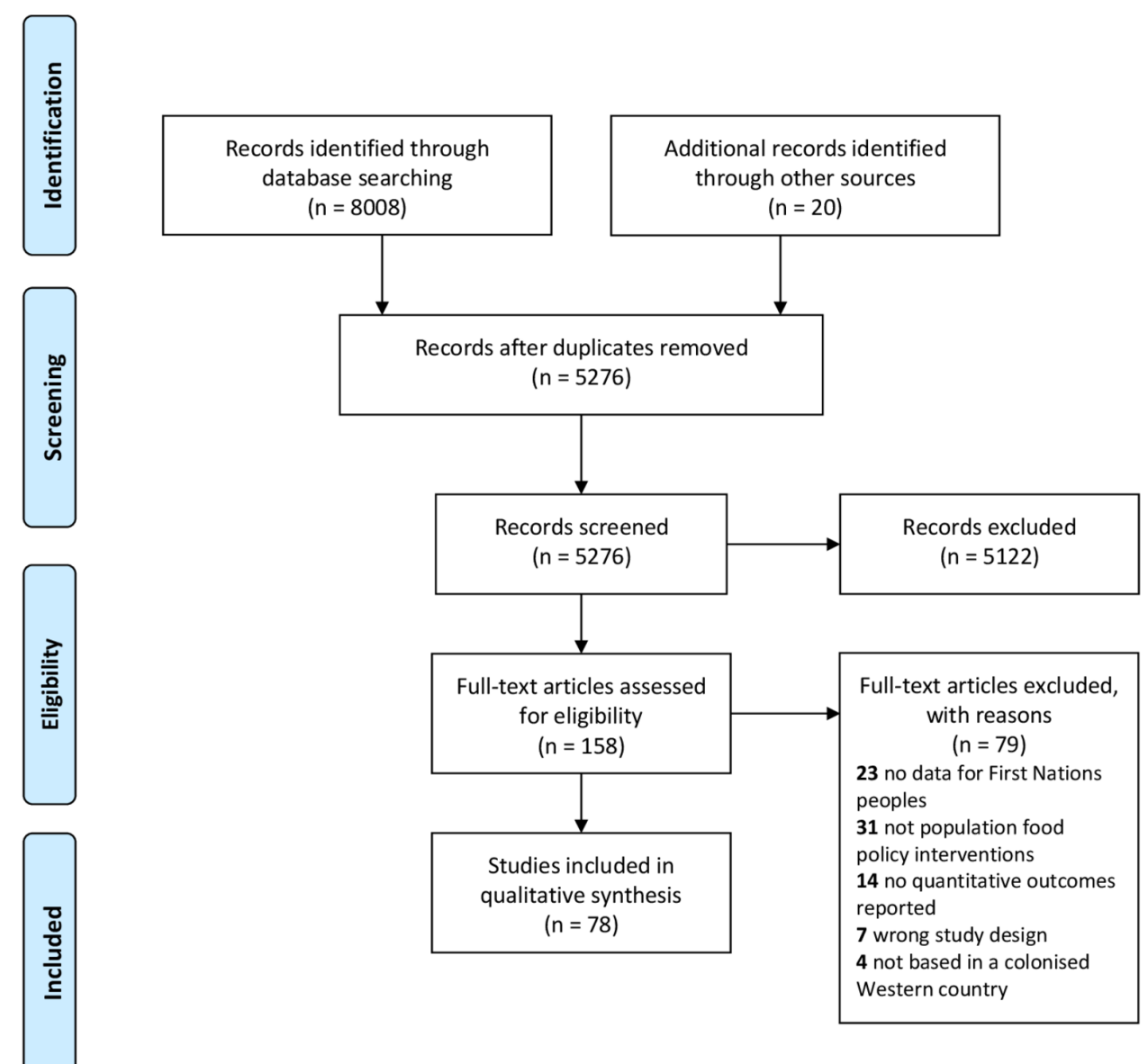

Figure 1 PRISMA flow chart of included and excluded articles. PRISMA, Preferred Reporting Items for Systematic Reviews and Meta-Analyses.

The review included 13 randomised controlled trials (RCTs), 42 non-randomised, quasi-experimental studies, 11 natural experiments, 11 cross-sectional surveys and 1 case-control study. Seven of the 78 studies were considered high quality. Most $(n=42)$ were assessed as moderate quality and 29 were rated low quality. The quality assessment components most frequently lacking were blinding, a control group, follow-up, reliability of measures and statistical analysis (particularly power calculations to detect an impact in Indigenous populations).

Change in self-reported food or nutrient intake was the most common outcome measure, included in approximately half $(\mathrm{n}=39)$ of all studies. A further 17 studies evaluated dietary change via store turnover or sales. Twenty-seven studies included anthropometric outcomes, 16 included biochemical markers and 15 included health outcomes (eg, dental caries, diabetes prevalence, birth outcomes). Five studies evaluated behavioural responses to food labels.

\section{Outcomes according to NOURISHING framework categories Nutrition labels}

Five articles evaluated behavioural responses to food labels. ${ }^{18-22}$ There were differences between Indigenous (Māori) and non-Indigenous (Pākehā) Peoples of Aotearoa (New Zealand) in the ability to use and interpret the Nutrition Information Panel and industry-led per cent dietary intake labels, with Māori participants less able to use these labels to determine whether a food was healthy. Interpretive labelling systems, such as traffic lights and health stars, were better understood for both Māori and Pākehā groups, although disparities remained. ${ }^{18}$ One RCT compared the Nutrition Information Panel, Traffic Light and Health Star rating systems among Māori and Pākehā and found no significant differences in food purchases between the labelling groups. ${ }^{22}$ Interestingly, among frequent label-users, interpretive systems led to more healthy food choices compared with when using the nutrition information panel, a result that did not vary between Māori and Pākehā. ${ }^{22}$ However, in a second post hoc analysis of the same trial, there was no significant difference (among Māori participants) in the nutritional value between foods/beverages purchased after viewing the interpretive label compared with products whose labels were viewed but not purchased..$^{21}$ In an Australian study, fouryears after the front-of-pack Health Star Rating System was implemented, Indigenous participants were significantly more likely than non-Indigenous participants to indicate this labelling system influenced their purchasing decisions. ${ }^{20}$ The only study to compare generic and culturally tailored food labels found that while both labels significantly increased selection of the healthier food item, the culturally tailored label was 


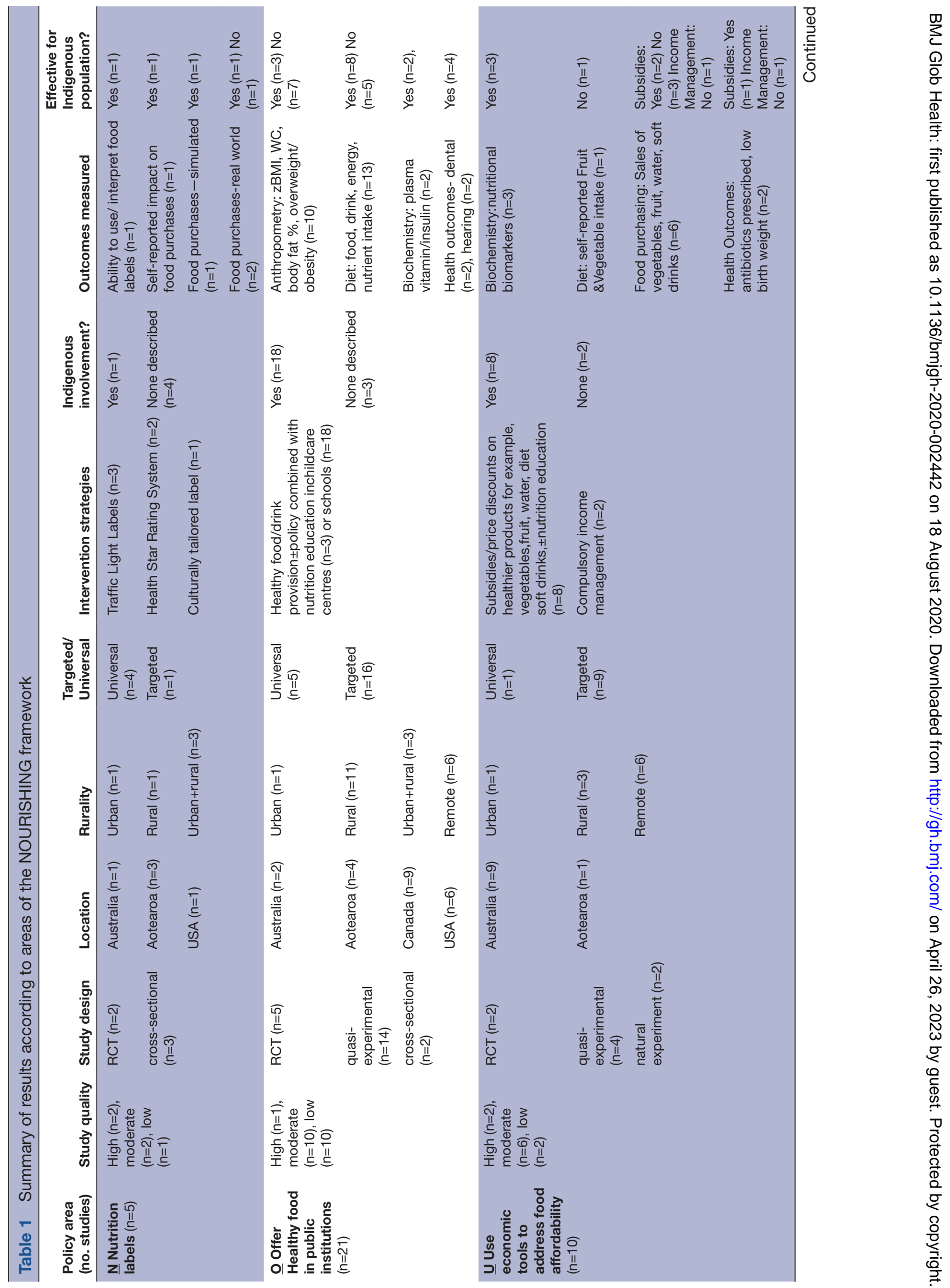




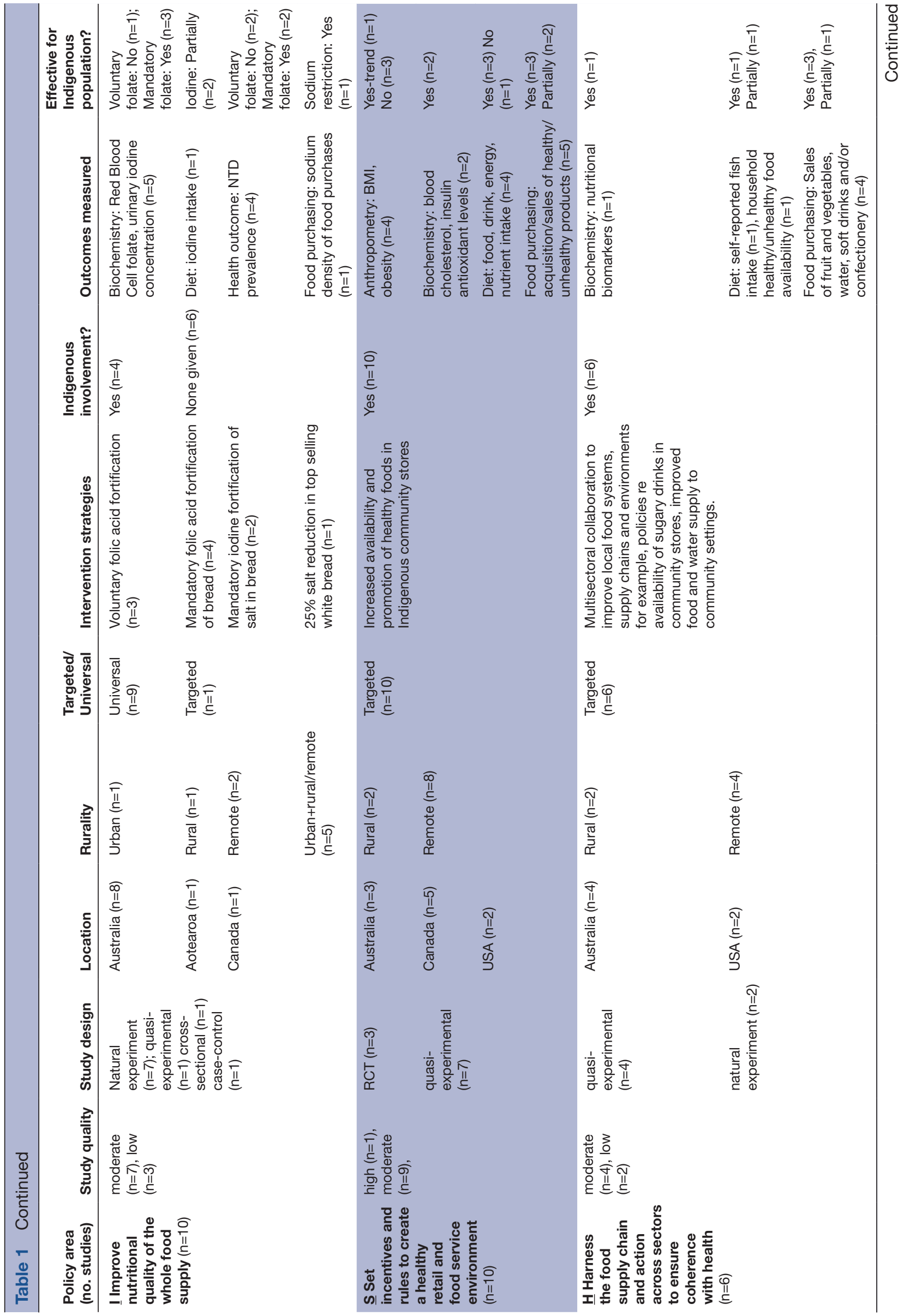

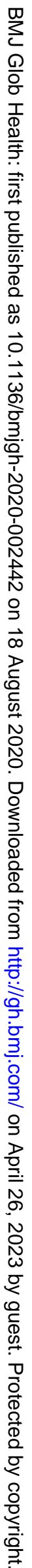




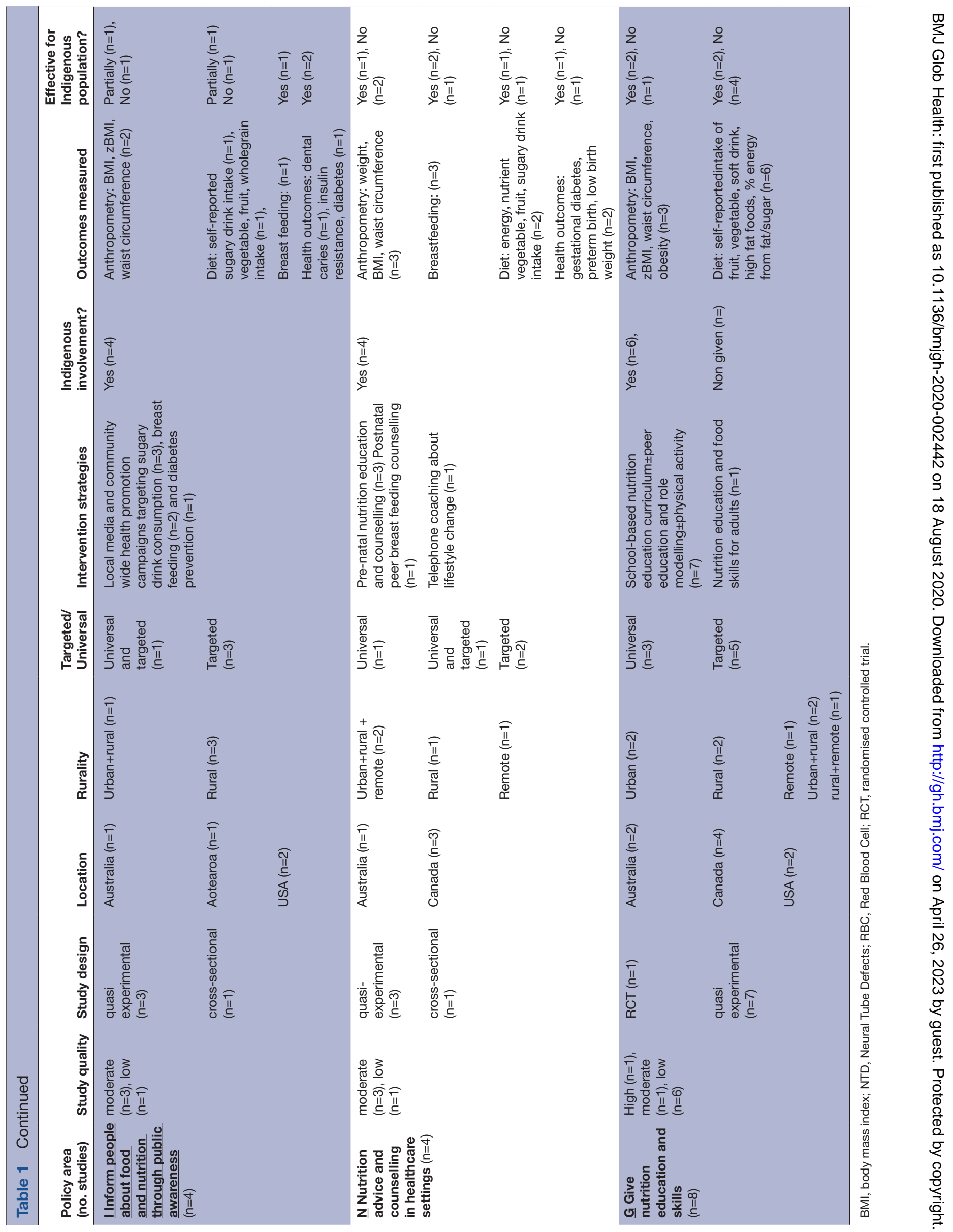


more effective than the generic label and significantly increased the price Indigenous shoppers were willing to pay for a healthy item. ${ }^{19}$

\section{Offer healthy food and set standards in public institutions}

Twenty-one articles described the impact of offering healthy food in schools or childcare centres. The majority $(n=13)$ of studies evaluating healthy food provision were undertaken in primary/elementary schools. ${ }^{26-38}$ Most of these were multicomponent interventions combining healthy food provision within school curriculum or other nutrition education activities. Ten studies measured anthropometric outcomes among Indigenous students, with most finding no significant difference in body mass index (BMI) postintervention, ${ }^{23}{ }^{25}$ however, one RCT reported a $10 \%$ reduction in the prevalence of overweight among Indigenous children. ${ }^{29}$ Two studies undertaken in general school populations with mixed ethnicities reported favourable effects on obesity prevalence, including Indigenous children. ${ }^{32} 37$ Studies evaluating dietary outcomes for Indigenous children frequently reported reductions in consumption of high fat/high sugar foods and beverages, ${ }^{26-29}$ and/or improvements in food group and nutrient intake. ${ }^{24} 3940$ A low-quality evaluation of a fruit provision programme for Indigenous children $(n=12)$ observed improvements in vitamin $C$ status and hearing tests following the intervention. ${ }^{33} 34$ Positive oral health outcomes were reported for Indigenous children attending childcare centres or schools with 'water only' policies. ${ }^{31} 37$ While another study demonstrated significant reductions in sugar-sweetened beverage consumption and plasma insulin levels among Indigenous high school students following the introduction of a nutrition policy, with insulin levels for males reaching parity with the non-Indigenous comparison group after three years. ${ }^{43}$

\section{Use economic tools to address food affordability}

Eight studies evaluated the impact of subsidising foods and beverages such as fruit, vegetables, bottled water and diet drinks, ${ }^{4-51}$ predominantly in rural/remote Australia. Findings suggests fruit and vegetable subsidies can improve fruit and vegetable intake $e^{48}$ and nutritional status ${ }^{44}$ in rural and remote communities. A high-quality RCT in remote Australian stores, where approximately $95 \%$ of the population was Indigenous, demonstrated that a $20 \%$ price discount on fruit and vegetables increased purchases by $12.7 \%$ during the discount period and by $19.8 \%$ after the discount was removed ${ }^{48}$ The evidence for price discounts on water and diet drinks was less clear. Most evaluations did not demonstrate significant changes in beverage consumption. ${ }^{480}$ Key factors determining the success of subsidy programmes, according to these studies, were the magnitude and promotion of the discount. ${ }^{49} 50$ The only study undertaken in an urban (Indigenous and nonIndigenous) population was unable to demonstrate a beneficial effect of price discounts on healthy foods for
Indigenous participants, with authors citing that this may have been due to high attrition. ${ }^{47}$ An adverse outcome observed in some studies of food subsidy programmes was a concurrent increase in overall calories purchased. ${ }^{481}$

Another economic intervention evaluated was compulsory income management. This Australian government intervention, which initially required suspension of the Racial Discrimination Act, quarantined 50\% of Indigenous people's social security payments for essential items including groceries. Two studies independently evaluated the impact of this government policy on store sales ${ }^{52}$ and birth outcomes. ${ }^{53}$ Both studies, which received ethical approval, were undertaken after the Racial Discrimination Act had been reinstated. The intervention had no beneficial effect on fruit, vegetable and soft drink purchasing ${ }^{52}$ while exposure to the income management policy reduced birth weights by over $100 \mathrm{~g}$ and increased the probability of low birth weight by $30 \% .{ }^{53}$

\section{Improving the nutritional quality of the food supply}

Nine studies evaluated the impact of food fortification. ${ }^{54-62}$ Australian evaluations demonstrated that voluntary folic acid fortification failed to improve folate status among Indigenous women and widened the gap in prevalence of neural tube defects (NTD) between Indigenous and non-Indigenous infants. ${ }^{55} 56$ Conversely, mandatory folic acid fortification was associated with significant reductions in folic acid deficiency among Indigenous women and marked reductions in NTD.$^{57} 62$ The most comprehensive study reported a $74 \%$ fall in NTD among Indigenous infants following mandatory fortification, almost completely closing the gap between Indigenous and non-Indigenous infants. ${ }^{59}$ Evaluation of mandatory bread fortification with iodine in Australia and Aotearoa demonstrated improvements in iodine intake ${ }^{60}$ and urinary iodine concentration ${ }^{61}$ among Indigenous women; however, pregnant women remained at risk of iodine deficiency. ${ }^{6061}$

Food reformulation is another intervention to improve the quality of the food supply. One study evaluated the impact of a $25 \%$ salt reduction in a top-selling bread in Indigenous stores in remote Australia. ${ }^{63}$ The intervention did not affect overall sales but resulted in a reduction in the sodium density of all foods purchased, which reached statistical significance when one control store was removed from the analysis.

Set incentives and rules to create a healthy retail and food service environment

Ten store-based interventions aimed to increase the availability and visibility of healthier options within Indigenous communities. ${ }^{64-73}$ Many of these also included health information/education activities within the store or in the broader Indigenous community. The Canadian Healthy Foods North study reported improvements in micronutrient intake, ${ }^{64}{ }^{71}$ reduced energy intake ${ }^{71}$ and decreased consumption of targeted high fat and sugar products among Inuit and Inuvialuit communities. ${ }^{69}$ 
Other North American studies found more frequent exposure to store-based interventions was associated with increased healthy food acquisition among Indigenous Peoples, ${ }^{656768}$ while another observed reduced unhealthy food acquisition among Indigenous overweight participants. ${ }^{70}$ The Australian studies reported a positive impact of store-based interventions on purchasing of fruit and vegetables and reductions in unhealthy foods and beverages, as a proportion of total energy purchased in Indigenous communities. ${ }^{6673}$ No studies were able to demonstrate statistically significant changes in BMI, ${ }^{67} 687072$ however, one observed a downward trend in BMI in favour of the store-based intervention and found higher intervention exposure was associated with significant reductions in BMI and improvements in overweight/obesity among Navajo Indigenous Peoples. ${ }^{67}$ Furthermore, an Australian study found improvements in biochemical markers of chronic disease risk, despite no changes in obesity prevalence for participants living in remote Indigenous communities. ${ }^{72} 73$

Harness the food supply chain and action across sectors to ensure coherence with health

Six studies evaluated multisectoral interventions aimed at improving food and/or beverage supply chains. ${ }^{90-95}$ Evidence from Australian remote Indigenous communities suggests community-driven and multisectoral collaboration to improve local food systems, supply chains and environments, can increase sales of fruit, vegetables and water and reduce sales of confectionary and sugarsweetened beverages. ${ }^{91} 929495$ Furthermore, the two North American studies demonstrated harnessing Indigenous and non-Indigenous food systems to deliver healthy food directly to community settings, such as schools or community centres, can increase intake of healthy foods (including traditional foods) and reduce food insecurity among Indigenous Peoples. ${ }^{90} 93$

\section{Inform people about food and nutrition through public awareness}

Providing nutrition information was a component of many interventions included in this review, however, four studies had public awareness campaigns as their primary intervention. ${ }^{74-77}$ These included community-wide, multicomponent health promotion campaigns, incorporating local media and community education, while encouraging food system/environmental change ${ }^{75-77}$ One of these studies in a Māori community in Aotearoa, who were at high risk of diabetes, observed significant reductions in the prevalence of insulin resistance among Māori women ${ }^{75}$ while others reported improvements in breast feeding and reduced dental caries among Native American infants after a community educational intervention. ${ }^{76} 77$ Another study compared Indigenous Peoples' responses to a universal and a culturally adapted and targeted media campaign, suggesting the targeted campaign was more effective than the universal campaign at reducing sugar-sweetened beverage consumption, although the results did not reach statistical significance. ${ }^{74}$

\section{Nutrition advice and counselling}

Four studies evaluated individual nutrition education and counselling services. ${ }^{78-81}$ Three focused on improving the health of pregnant women and infants through maternal education and counselling during the prenatal/postnatal period. ${ }^{78-80}$ All studies reported positive results for breast feeding, with the largest improvements from a programme employing Indigenous peer-counsellors to provide targeted breastfeeding advice and support. ${ }^{79}$ The universal Canadian Prenatal Nutrition Programme was also associated with improved breastfeeding duration and reduced risk of low birth weight among First Nations infants, however, excess weight gain during pregnancy and increased numbers of large for gestational age babies were noted as unintended consequences. ${ }^{80}$ The other intervention was a universally available telephone lifestyle coaching service that was culturally tailored to increase participation of Australian Indigenous People. ${ }^{81}$ The evaluation reported significant improvements in selfreported BMI, waist circumference and dietary indicators with no significant differences between improvements made by Indigenous and non-Indigenous participants. ${ }^{81}$

\section{Give nutrition education and skills}

Eight studies evaluated nutrition education programmes. ${ }^{82-89}$ Most $(\mathrm{n}=6)$ were of short duration, had small sample sizes and relied on self-reported dietary outcomes. A high quality evaluation of a school-based peer education programme in which older students mentored younger students about nutrition, physical activity and self-esteem found a reduction in waist circumference among Indigenous children that was almost double that of non-Indigenous children. ${ }^{88}$ Cultural tailoring of this programme for Indigenous schools resulted in significant reductions in zBMI and waist circumference among participants, while zBMI increased in the control group. ${ }^{87}$ Other studies reported short-term increases in fruit and vegetable intake among Indigenous children and adults following nutrition education, ${ }^{83} 86$ however, long-term evaluation of a school-based programme was not able to demonstrate significant improvements in children's diets. $^{84}$

\section{Involvement of Indigenous Peoples}

Sixty of the 78 publications described the involvement of Indigenous Peoples in intervention design or other cultural considerations in the research (see online supplementary table 2), while 18 studies did not provide any details about Indigenous engagement. Engagement was variously described through gaining permission from local Elders and organisations; involvement in programme initiation, design, implementation, staffing, evaluation; and, in some cases, translation of outcomes.

\section{DISCUSSION}

This is the first multicountry systematic review to examine the impact of food and nutrition policy actions on Indigenous Peoples. We found 78 articles, published over the 
last 20 years in four Western colonised countries. We identified food policy actions across all but one of the areas (marketing) where governments need to take action to promote healthy diets according to the NOURISHING framework. Although the populations included in this review have different lands, ethnicity, culture, language and food practices, they all experience health disparities underpinned by social inequity and the ongoing impact of colonisation. Many of the policy actions included in this review have demonstrated significant improvements in a range of nutrition and health outcomes for Indigenous Peoples; however, many evidence gaps remain.

A salient finding of this review is population-wide policies, including mandatory food fortification, reformulation and interpretive front of pack labelling systems, have the potential to reduce health inequities between Indigenous and non-Indigenous Peoples: when universally applied. This is exemplified by mandatory folic acid fortification in Australia, which almost completely closed the gap in Neural Tube Defects between Indigenous and non-Indigenous infants, ${ }^{59}$ while voluntary fortification widened this gap. ${ }^{556}$ The studies in our synthesis, including one on sodium reduction ${ }^{63}$ suggest policies to improve the nutritional quality of the food supply can be particularly beneficial for Indigenous Peoples; however, these are not consistently applied across countries. Australia, Canada and the USA all have mandatory folic acid fortification policies in place; however, in Aotearoa folic acid fortification remains voluntary. Similarly, Australia, Aotearoa and Canada mandate iodine fortification while the USA does not. None of the countries included in this review have implemented mandatory sodium reduction targets. With respect to sodium restriction, given the lack of food industry action when food reformulation targets are voluntary ${ }^{96}{ }^{97}$ mandatory food reformulation policies have greater potential to reduce health inequities.

We found that interpretive food labelling systems may be more effective at facilitating healthy food purchasing decisions compared with the mandatory nutrition information panel. ${ }^{1822}$ While the Health Star Rating front-ofpack interpretive labelling system has been implemented in Australia and Aotearoa, it remains as a voluntary policy. Initial evaluation of this labelling system has yielded promising results for self-reported influence on purchasing decisions among Indigenous Peoples ${ }^{20}$; however, the study was narrowly focused (two survey questions on perceived influence), limiting the generalisability of the findings. Furthermore, the Health Star Rating system, in its current form, has been criticised for being reductionist and facilitating marketing of processed foods; and is currently under review. ${ }^{98} 99$ Thus, while our findings support interpretive front-of-pack labels per se, we do not seek to endorse the specific algorithms used for this labelling system.

Previous systematic reviews found school nutrition policies, combined with nutrition education and family engagement, are among the most promising obesity prevention interventions. ${ }^{100} 101$ Our findings confirm comprehensive school and child-care centre-based policies are also a promising approach for Indigenous children. Many of the studies reviewed reported improvements in nutrition-related outcomes, although methodological quality varied. Three interventions with a focus on reducing sugar-sweetened beverages in children's settings demonstrated statistically significant improvements in health outcomes for Indigenous children, including dental health ${ }^{2531}$ and insulin sensitivity, ${ }^{43}$ suggesting a pro-equity effect. Although most schoolbased interventions did not change BMI, those studies that evaluated BMI as an outcome were of relatively short duration. The one long-term evaluation of a populationwide school health promotion programme demonstrated statistically significant improvements in childhood obesity rates across Indigenous and non-Indigenous groups. ${ }^{37}$

Food retail and supply chain interventions to improve local food and beverage environments in rural and remote Indigenous communities were consistently effective across included studies. Consistent with the results of a recent review of retail food environment interventions, ${ }^{102}$ all studies targeting local food retail and supply chains within Indigenous communities were able to demonstrate improvements in at least one outcome including dietary quality, healthy food purchasing, food security, nutrition or chronic disease biomarkers, and one reported a downward trend in mean BMI. ${ }^{67}$

Furthermore, the use of store sales data to evaluate the impact of remote Indigenous community store interventions in Australia provided objective evidence for multisectoral food system interventions to increase purchasing of fruits and vegetables, and decrease purchasing of confectionary and sugar-sweetened beverages. ${ }^{66} 919294$ Critically, all of these studies reported strong Indigenous involvement and ownership of local Indigenous communities in intervention development, implementation and/or evaluation. Conversely, interventions targeting Indigenous Peoples implemented without Indigenous leadership or consultation, such as income management, do not improve nutrition outcomes and may be harmful. ${ }^{5253}$ It remains unclear as to whether the positive findings from remote communities can be translated to Indigenous Peoples living in urban areas. The only storebased intervention undertaken in urban and regional areas was not sufficiently powered to detect geographical subgroup differences. ${ }^{47}$

Food and beverage pricing policies are frequently proposed as an effective strategy to improve population diets and health equity. ${ }^{103-106}$ Our review indicates subsidies/price discounts, when of sufficient magnitude and adequately promoted, can be effective at improving fruit and vegetable consumption, ${ }^{48}{ }^{51}$ nutritional status and children's health outcomes ${ }^{44-46}$ in Aboriginal communities in rural and remote Australia. However, concurrent strategies are required to prevent the concomitant increase in purchasing of energy-dense foods and beverages observed in these studies. ${ }^{48}{ }^{93}$ Further, while the 
evidence was strong for targeted fruit and vegetable subsidies, we did not identify any studies examining the impact of universal food or beverage taxes on Indigenous Peoples' food purchases, health or well-being. Evaluation of the sugar-sweetened beverage tax in Mexico revealed reductions in sugar-sweetened beverage purchasing were greatest among lower socioeconomic households, ${ }^{107} 108$ but the impact on the Indigenous Peoples of Mexico has not been analysed. Policies that increase the price of sugar sweetened beverages are in place in some Indigenous community stores in remote Australia ${ }^{109}$ and the Navajo Nation (Native American territory) has introduced a $2 \%$ tax on unhealthy foods and beverages and removed a $5 \%$ sales tax on fruit, vegetables and water. ${ }^{110}$ It will be important to evaluate these policies for their impact on Indigenous Peoples.

There is convincing evidence that exposure to unhealthy food advertising influences children's food intake. ${ }^{111} 112$ Mandatory restrictions on marketing of unhealthy foods and beverages are likely to be effective across socioeconomic groups. ${ }^{113} 114$ However, we found no evaluations of the impact of food advertising restrictions on Indigenous Peoples. This is particularly concerning as it has been estimated that Canadian First Nations children are exposed to one food advertisement every 5 min. ${ }^{28}$ Furthermore, there is evidence from Aotearoa suggesting Māori children have higher rates of exposure to food, alcohol and gambling marketing compared with non-Māori children. ${ }^{115-117}$ Policy action and evaluation in this area is another evidence gap that needs to be closed.

Public information campaigns, lifestyle advice and nutrition education are often not the preferred strategy of public health advocates as they target individual behaviour change rather than the structural determinants of food choice. ${ }^{13} 118$ The evidence in this review indicates a place for educational interventions, especially when implemented concurrently with approaches targeting food systems and environments. The best results were from studies of community-wide, culturally relevant nutrition information and education programmes with multiple components, which showed improvements in insulin sensitivity, BMI and dental health. ${ }^{75-77818788}$ Oneto-one nutrition education and counselling, especially using an Indigenous peer education approach, effectively improved breastfeeding rates, BMI and waist circumference. ${ }^{79} 8788$ Previous reviews have also found community nutrition education and health promotion campaigns can be effective when implemented alongside changes to the food environment and with high levels of community involvement. ${ }^{14119-121}$

Most studies included in this review were of moderate methodological quality, with only 7 out of 78 were assessed as high quality. Although the JBI quality assessment tools were useful as they allowed different study designs, they are intended for clinically-based studies rather than complex population-based or 'real-world' interventions. The appropriateness of quality appraisal tools for multicomponent public health interventions is frequently questioned. ${ }^{120} 122$ Furthermore, the aspects of 'quality' included in existing tools are constructed through a Western ontological lens and are likely entirely different from what is considered high quality research from Indigenous Peoples' perspectives. ${ }^{123}$

Many of the studies in this review reported the ways in which researchers engaged with Indigenous communities in intervention design and evaluation. Indigenous leadership; community engagement and involvement; participatory approaches; trust and relationships; incorporation of cultural knowledge; culturally safe approaches and translation of findings and benefits back to participating communities are among the features consistently identified as critical to the success of health promotion and public health research in Indigenous communities. ${ }^{124} 125$ However, despite the increasing volume of research conducted in the food and nutrition policy field (one-third of all studies were published in the last fouryears), there still exists no universally accepted tool for appraising public health and health promotion interventions for Indigenous Peoples. Indigenous Peoples have a long history of critiquing health research and several Indigenous-led research groups have piloted tools for appraising health promotion research in Indigenous contexts. ${ }^{123} 126$ There is clearly the need for further rigorous evaluation research into food and nutrition interventions in Indigenous populations, including the differential impact of population-wide policy actions. However, equally important is the development of research protocols and quality assessment tools that value both Indigenous and Western ways of knowing.

\section{Strengths and limitations}

This review makes an important contribution to the evidence for both population-wide and Indigenousspecific food and nutrition policy action. It included an extensive search strategy, incorporating international peer-reviewed and grey literature and broad inclusion criteria to ensure a diverse range of Indigenous populations, policy actions, outcomes and study designs. A further strength of this review is the inclusion of Indigenous researchers on the review team who were involved in every step of the process including protocol design, study screening, data extraction and quality assessment.

We also note some limitations. Like all systematic reviews, our synthesis may be limited by publication bias as unsuccessful interventions are less likely to appear in the literature. We attempted to reduce this risk by including grey literature and contacting experts in the field for unpublished reports. Our review only included studies published in English from Western colonised countries, thus policies implemented in Indigenous populations in non-English-speaking, low-income and middle-income countries have not been included. It is possible this systematically excluded potentially relevant studies from Latin America, a region with increasing implementation of regulatory food and nutrition policies. ${ }^{127}$ However, within the four countries included 
in the current review, the great diversity of the Indigenous populations represented means that generalisability is not possible. Furthermore, Indigenous peoples worldwide represent a much broader range of nations, language groups, cultures, gender identities and food system contexts than the populations included in this review. There is a need for further research to capture the full range of Indigenous experiences. Despite these limitations, we draw some high-level conclusions and implications for policy and practice.

\section{CONCLUSION}

Our findings suggest that to improve nutrition-related health outcomes for Indigenous Peoples, both targeted and universal policy actions are required. Targeted approaches should be community-directed, participatory, culturally relevant, multifaceted interventions combining strategies to improve food affordability, healthy food environments and nutrition knowledge and skills. To promote equity, universal approaches should change the structures and systems in which populations access food, which for Indigenous Peoples, have been severely disrupted by colonisation. Promising approaches include food pricing policies that incentivise healthy products and disincentive unhealthy products; mandatory rather than voluntary food reformulation targets and interpretive food labelling systems; and restrictions on junk food marketing to children, however, further research is required to confirm this. Rigorous designs with sufficient statistical power, careful monitoring and evaluation are required to determine the differential impact of population-wide policy actions on Indigenous Peoples, particularly in urban areas.

Acknowledgements We respectfully acknowledge the Indigenous Peoples in the articles included in this review: Aboriginal and Torres Strait Islander (Australia), Māori (Aotearoa/New Zealand), First Nations, Inuit and Inuvialuit (Canada) and American Indian and Alaskan Native Peoples. This research took place on the lands of the Wurundjeri, Wathaurong, Yorta Yorta and Awabakal Peoples of Australia. ML is a Ngiyampaa man, TW is a Yorta Yorta man and ME is a Gunditjmara/Mutti Mutti woman.

Contributors $\mathrm{JB}$ conceptualised the study and developed the protocol in consultation with KB and ME. JB, ML and TW screened and selected the studies, extracted and analysed data. JB drafted the initial manuscript, TW, ML, KB and ME provided feedback on initial drafts and further revised the manuscript. All authors read and approved the final manuscript.

Funding JB is supported by an Alfred Deakin Postdoctoral Research Fellowship. KB is supported by a Heart Foundation Future Leader Fellowship (102047). This study was funded by the Victorian Health Promotion Foundation (VicHealth).

Competing interests None declared.

Patient and public involvement Patients and/or the public were involved in the design, or conduct, or reporting, or dissemination plans of this research. Refer to the Methods section for further details.

Patient consent for publication Not required.

Provenance and peer review Not commissioned; externally peer reviewed.

Data availability statement All data relevant to the study are included in the article or uploaded as online supplementary information. Details of included studies are included in online supplementary file 2 .

Open access This is an open access article distributed in accordance with the Creative Commons Attribution Non Commercial (CC BY-NC 4.0) license, which permits others to distribute, remix, adapt, build upon this work non-commercially, and license their derivative works on different terms, provided the original work is properly cited, appropriate credit is given, any changes made indicated, and the use is non-commercial. See: http://creativecommons.org/licenses/by-nc/4.0/.

\section{REFERENCES}

1 United Nations. United Nations Declaration on the Rights of Indigenous Peoples, 2007. Available: http://www.un.org/esa/ socdev/unpfii/documents/DRIPS_en.pdf

2 Anderson I, Robson B, Connolly $\bar{M}$, et al. Indigenous and tribal peoples' health (the Lancet-Lowitja Institute global collaboration): a population study. The Lancet 2016;388:131-57.

3 Carson B, Dunbar T, Chenhall RD, et al. Social determinants of Indigenous health. Allen \& Unwin, 2007.

4 Kuhnlein HV. Food system sustainability for health and well-being of Indigenous peoples. Public Health Nutr 2015;18:2415-24.

5 Kuhnlein $\mathrm{H}$, Erasmus B, Creed-Kanashiro $\mathrm{H}$, et al. Indigenous peoples' food systems for health: finding interventions that work. Public Health Nutr 2006;9:1013-9.

6 Levkoe C, Ray L, Mclaughlin J. The Indigenous food circle: reconciliation and resurgence through food in northwestern Ontario. J Agric Food Syst Community Dev 2019;9:1-14.

7 Lee A, Ride K. Review of nutrition among Aboriginal and Torres Strait Islander people. Perth: Australian Indigenous HealthlnfoNet; 2018.Kshatriya GK, Acharya SK. triple burden of obesity, undernutrition, and cardiovascular disease risk among Indian tribes. PLoS One 2016;11.

8 Kshatriya GK, Acharya SK. Triple burden of obesity, undernutrition, and cardiovascular disease risk among Indian tribes. PLOS One 2016;11:e0147934.

9 Forouzanfar MH, Afshin A, Alexander LT, et al. Global, regional, and national comparative risk assessment of 79 behavioural, environmental and occupational, and metabolic risks or clusters of risks, 1990-2015: a systematic analysis for the global burden of disease study 2015. Lancet 2016;388:1659-724.

10 International Food Policy Research Institute. Global nutrition report. Washington DC: International Food Policy Research Institute, 2017.

11 Willett W, Rockström J, Loken B, et al. Food in the Anthropocene: the EAT-Lancet Commission on healthy diets from sustainable food systems. Lancet 2019;393:447-92.

12 Food and Agricultural Organization of the United Nations,, World Health Organization. Second International Conference on nutrition, conference outcome document: framework for action. Rome: FAO and $\mathrm{WHO}, 2014$.

13 Backholer K, Beauchamp A, Ball K, et al. A framework for evaluating the impact of obesity prevention strategies on socioeconomic inequalities in weight. Am J Public Health 2014;104:e43-50.

14 Beauchamp A, Backholer K, Magliano D, et al. The effect of obesity prevention interventions according to socioeconomic position: a systematic review. Obes Rev 2014:15:541-54.

15 Hawkes C, Jewell J, Allen K. A food policy package for healthy diets and the prevention of obesity and diet-related noncommunicable diseases: the NOURISHING framework. Obes Rev 2013;14 Suppl 2:159-68.

16 Welch V, Petticrew M, Tugwell P, et al. PRISMA-Equity 2012 extension: reporting guidelines for systematic reviews with a focus on health equity. PLoS Med 2012;9:e1001333.

17 Tufanaru C, Munn Z, Aromataris E, et al. Chapter 3: Systematic reviews of effectiveness. In: Aromataris E, ed. Joanna Briggs Institute reviewer's manual. Adelaide: The Joanna Briggs Institute, 2017.

18 Gorton D, Ni Mhurchu C, Chen M-H, et al. Nutrition labels: a survey of use, understanding and preferences among ethnically diverse shoppers in New Zealand. Public Health Nutr 2009;12:1359-65.

19 Gustafson CR, Prate MR. Healthy food labels tailored to a high-risk minority population more effectively promote healthy choices than generic labels. Nutrients 2019;11:2272.

20 Heart Foundation of Australia. Report on the monitoring of the implementation of the Health Star Rating system in the first four years of implementation: June 2014 to June 2018. Canberra: Commissioned by the Commonwealth Department of Health, 2019.

21 Ni Mhurchu $\mathrm{C}$, Eyles $\mathrm{H}$, Jiang $\mathrm{Y}$, et al. Do nutrition labels influence healthier food choices? Analysis of label viewing behaviour and subsequent food purchases in a labelling intervention trial. Appetite 2018;121:360-5

22 Ni Mhurchu C, Volkova E, Jiang Y, et al. Effects of interpretive nutrition labels on consumer food purchases: the Starlight randomized controlled trial. Am J Clin Nutr 2017;105:695-704. 
23 Davis SM, Myers OB, Cruz TH, et al. Chile: outcomes of a group randomized controlled trial of an intervention to prevent obesity in preschool Hispanic and American Indian children. Prev Med 2016;89:162-8.

24 Gagné D, Blanchet R, Vaissière Émilie, et al. Impact of a childcare centre nutrition program on nutrient intakes in Nunavik Inuit children. Can J Diet Pract Res 2013;74:e311-7.

25 Rush E, Obolonkin V, Young L, et al. Under 5 Energize: tracking progress of a preschool nutrition and physical activity programme with regional measures of body size and dental health at age of four years. Nutrients 2017;9:456.

26 Caballero B, Clay T, Davis SM, et al. Pathways: a school-based, randomized controlled trial for the prevention of obesity in American Indian schoolchildren. Am J Clin Nutr 2003;78:1030-8.

27 Himes JH, Ring K, Gittelsohn J, et al. Impact of the pathways intervention on dietary intakes of American Indian schoolchildren. Prev Med 2003;37:S55-61.

28 Paradis G, Lévesque L, Macaulay AC, et al. Impact of a diabetes prevention program on body size, physical activity, and diet among Kanien'keha:ka (Mohawk) children 6 to 11 years old: 8-year results from the Kahnawake Schools Diabetes Prevention Project. Pediatrics 2005;115:333-9.

29 Story M, Hannan PJ, Fulkerson JA, et al. Bright Start: description and main outcomes from a group-randomized obesity prevention trial in American Indian children. Obesity 2012;20:2241-9.

30 Rush E, Reed P, McLennan S, et al. A school-based obesity control programme: Project Energize. two-year outcomes. Br J Nutr 2012;107:581-7.

31 Thornley S, Marshall R, Reynolds G, et al. Low sugar nutrition policies and dental caries: a study of primary schools in South Auckland. J Paediatr Child Health 2017;53:494-9.

32 Vogeltanz-Holm N, Holm J. Changes in body mass index during a 3-year elementary school-based obesity prevention program for American Indian and white rural students. Health Educ Behav 2018;45:277-85

33 Jones R, Smith F. Are there health benefits from improving basic nutrition in a remote Aboriginal community? Aust Fam Physician 2006;35:453-4.

34 Jones R, Smith F. Fighting disease with fruit. Aust Fam Physician 2007;36:863-4

35 Kakekagumick KE, Naqshbandi Hayward M, Harris SB, et al. Sandy Lake health and diabetes project: a community-based intervention targeting type 2 diabetes and its risk factors in a first nations community. Front Endocrinol 2013;4.

36 Hanbazaza MA, Triador L, Ball GDC, et al. The impact of school gardening on Cree children's knowledge and attitudes toward vegetables and fruit. Can J Diet Pract Res 2015;76:133-9.

37 Rush E, McLennan S, Obolonkin V, et al. Project Energize: wholeregion primary school nutrition and physical activity programme; evaluation of body size and fitness 5 years after the randomised controlled trial. Br J Nutr 2014;111:363-71.

38 Triador L, Farmer A, Maximova K, et al. A school gardening and healthy snack program increased Aboriginal First Nations children's preferences toward vegetables and fruit. J Nutr Educ Behav 2015;47:176-80.

39 Saksvig BI, Gittelsohn J, Harris SB, et al. A pilot school-based healthy eating and physical activity intervention improves diet, food knowledge, and self-efficacy for native Canadian children. J Nutr 2005;135:2392-8.

40 Skinner K, Hanning RM, Metatawabin J, et al. Impact of a school snack program on the dietary intake of grade six to ten First Nation students living in a remote community in northern Ontario, Canada. Rural Remote Health 2012;12:2122.

41 Gates M, Hanning RM, Gates A, et al. A pilot comprehensive school nutrition program improves knowledge and intentions for intake of milk and milk alternatives among youth in a remote First Nation. $J$ Nutr Educ Behav 2013;45:455-9.

42 Gates M, Hanning RM, Gates A, et al. Assessing the impact of pilot school snack programs on milk and alternatives intake in 2 remote First Nation communities in northern Ontario, Canada. J Sch Health 2013;83:69-76.

43 Ritenbaugh C, Teufel-Shone NI, Aickin MG, et al. A lifestyle intervention improves plasma insulin levels among Native American high school youth. Prev Med 2003;36:309-19.

44 Black AP, Vally $\mathrm{H}$, Morris $\mathrm{P}$, et al. Nutritional impacts of a fruit and vegetable subsidy programme for disadvantaged Australian Aboriginal children. Br J Nutr 2013;110:2309-17.

45 Black AP, Vally $\mathrm{H}$, Morris $\mathrm{P}$, et al. High folate levels in Aboriginal children after subsidised fruit and vegetables and mandatory folic acid fortification. Aust N Z J Public Health 2014;38:241-6.
46 Black AP, Vally H, Morris PS, et al. Health outcomes of a subsidised fruit and vegetable program for Aboriginal children in northern New South Wales. Med J Aust 2013;199:46-50.

47 Blakely T, Ni Mhurchu C, Jiang Y, et al. Do effects of price discounts and nutrition education on food purchases vary by ethnicity, income and education? Results from a randomised, controlled trial. J Epidemiol Community Health 2011;65:902-8.

48 Brimblecombe J, Ferguson M, Chatfield MD, et al. Effect of a price discount and consumer education strategy on food and beverage purchases in remote Indigenous Australia: a stepped-wedge randomised controlled trial. Lancet Public Health 2017;2:e82-95.

49 Brown C, Laws C, Leonard D, et al. Healthy Choice Rewards: a feasibility trial of incentives to influence consumer food choices in a remote Australian Aboriginal community. Int J Environ Res Public Health 2019;16:112.

50 Ferguson M, O'Dea K, Holden S, et al. Food and beverage price discounts to improve health in remote Aboriginal communities: mixed method evaluation of a natural experiment. Aust $N Z J$ Public Health 2017;41:32-7

51 Lee A, Rainow S, Tregenza J, et al. Nutrition in remote Aboriginal communities: lessons from Mai Wiru and the Anangu Pitjantjatjara Yankunytjatjara Lands. Aust N Z J Public Health 2016;40:S81-8.

52 Brimblecombe JK, McDonnell J, Barnes A, et al. Impact of income management on store sales in the Northern Territory. Med J Aust 2010;192:549-54.

53 Doyle M-A, Schurer S, Silburn S. Do welfare restrictions improve child health? Estimating the causal impact of income management in the Northern Territory. Brisbane: Life Course Centre, University of Queensland, 2017.

54 Arbour L, Rupps R, MacDonald S, et al. Congenital heart defects in Canadian Inuit: is more folic acid making a difference? Alaska Med 2007;49:163-6.

55 Bower C, D'Antoine H, Stanley FJ. Neural tube defects in Australia: trends in encephaloceles and other neural tube defects before and after promotion of folic acid supplementation and voluntary food fortification. Birth Defects Res A Clin Mol Teratol 2009;85:269-73.

56 Bower C, Eades S, Payne J, et al. Trends in neural tube defects in Western Australia in Indigenous and non-Indigenous populations. Paediatr Perinat Epidemiol 2004;18:277-80.

57 Bower C, Maxwell S, Hickling S, et al. Folate status in Aboriginal people before and after mandatory fortification of flour for breadmaking in Australia. Aust N Z J Obstet Gynaecol 2016;56:233-7.

58 D'Onise K, McDermott RA, Leonard D, et al. Lack of folate improvement in high risk Indigenous Australian adults over an average of 6.5 years: a cohort study. Asia Pac J Clin Nutr 2012;21:431-9.

59 Hilder L. Neural tube defects in Australia, 2007-2011: before and after implementation of the mandatory folic acid fortification standard. Sydney: National Perinatal Epidemiology and Statistics Unit, University of New South Wales, 2016.

60 Mallard SR, Houghton LA. Public health policy to redress iodine insufficiency in pregnant women may widen sociodemographic disparities. Public Health Nutr 2014;17:1421-9.

61 Singh GR, Davison B, Ma GY, et al. lodine status of Indigenous and non-Indigenous young adults in the top end, before and after mandatory fortification. Med J Aust 2019;210:121-5.

62 Slagman A, Harriss L, Campbell S, et al. Low proportions of folic acid deficiency after introduction of mandatory folic acid fortification in remote areas of northern Queensland, Australia: a secondary health data analysis. Biomarkers 2019;24:684-91.

63 McMahon E, Webster J, Brimblecombe J. Effect of $25 \%$ Sodium Reduction on Sales of a Top-Selling Bread in Remote Indigenous Australian Community Stores: A Controlled Intervention Trial. Nutrients 2017;9:214.

64 Bains A, Pakseresht M, Roache C, et al. Healthy Foods North improves diet among Inuit and Inuvialuit women of childbearing age in Arctic Canada. J Hum Nutr Diet 2014;27 Suppl 2:175-85.

65 Bird Jernigan VB, Salvatore AL, Williams M, et al. A healthy retail intervention in native American convenience stores: the THRIVE community-based participatory research study. Am J Public Health 2019;109:e1-8.

66 Brimblecombe J, Ferguson M, McMahon E, et al. Reducing retail merchandising of discretionary food and beverages in remote Indigenous community stores: protocol for a randomized controlled trial. JMIR Res Protoc 2019;8:e12646.

67 Gittelsohn J, Kim EM, He S, et al. A food store-based environmental intervention is associated with reduced $\mathrm{BMI}$ and improved psychosocial factors and food-related behaviors on the Navajo nation. J Nutr 2013;143:1494-500.

68 Ho LS, Gittelsohn J, Rimal R, et al. An integrated multi-institutional diabetes prevention program improves knowledge and healthy 
food acquisition in northwestern Ontario First Nations. Health Educ Behav 2008;35:561-73.

69 Kolahdooz F, Pakseresht M, Mead E, et al. Impact of the Healthy Foods North nutrition intervention program on Inuit and Inuvialuit food consumption and preparation methods in Canadian Arctic communities. Nutr J 2014;13.

70 Mead EL, Gittelsohn J, Roache C, et al. A community-based, environmental chronic disease prevention intervention to improve healthy eating psychosocial factors and behaviors in Indigenous populations in the Canadian Arctic. Health Educ Behav 2013;40:592-602.

71 Pakseresht M, Kolahdooz F, Gittelsohn J, et al. Improving vitamin A and $D$ intake among Inuit and Inuvialuit in Arctic Canada: evidence from the Healthy Foods North study. J Epidemiol Community Health 2015;69:453-9.

72 Rowley KG, Daniel M, Skinner K, et al. Effectiveness of a community-directed 'healthy lifestyle' program in a remote Australian aboriginal community. Aust N Z J Public Health 2000;24:136-44.

73 Rowley KG, Su Q, Cincotta M, et al. Improvements in circulating cholesterol, antioxidants, and homocysteine after dietary intervention in an Australian Aboriginal community. Am J Clin Nutr 2001;74:442-8.

74 Browne J, MacDonald C, Egan M, et al. You wouldn't eat 16 teaspoons of sugar-so why drink it? Aboriginal and Torres Strait Islander responses to the LiveLighter sugary drink campaign. Health Promot J Austr 2019;30:212-8.

75 Coppell KJ, Tipene-Leach DC, Pahau HLR, et al. Two-Year results from a community-wide diabetes prevention intervention in a high risk Indigenous community: the Ngati and healthy project. Diabetes Res Clin Pract 2009;85:220-7.

76 Karanja N, Lutz T, Ritenbaugh C, et al. The TOTS community intervention to prevent overweight in American Indian toddlers beginning at birth: a feasibility and efficacy study. $J$ Community Health 2010;35:667-75.

77 Maupomé G, Karanja N, Ritenbaugh C, et al. Dental caries in American Indian toddlers after a community-based beverage intervention. Ethn Dis 2010;20:444-50.

78 Gray-Donald K, Robinson E, Collier A, et al. Intervening to reduce weight gain in pregnancy and gestational diabetes mellitus in Cree communities: an evaluation. CMAJ 2000;163:1247-51.

79 Martens PJ. The effect of breastfeeding education on adolescent beliefs and attitudes: a randomized school intervention in the Canadian Ojibwa community of Sagkeeng. J Hum Lact 2001;17:245-55.

80 Muhajarine $\mathrm{N}, \mathrm{Ng} \mathrm{J}$, Bowen A, et al. Understanding the impact of the Canada prenatal nutrition program: a quantitative evaluation. Can J Public Health 2012;103:S26-31.

81 Quinn E, O'Hara BJ, Ahmed N, et al. Enhancing the get healthy information and coaching service for Aboriginal adults: evaluation of the process and impact of the program. Int $J$ Equity Health 2017;16.

82 Frenn M, Malin S, Bansal N, et al. Addressing health disparities in middle school students' nutrition and exercise. J Community Health Nurs 2003;20:1-14.

83 Govula C, Kattelman K, Ren C. Culturally appropriate nutrition lessons increased fruit and vegetable consumption in American Indian children. Top Clin Nutr 2007;22:239-45.

84 Jimenez MM, Receveur O, Trifonopoulos M, et al. Comparison of the dietary intakes of two different groups of children (grades 4 to 6) before and after the Kahnawake schools diabetes prevention project. J Am Diet Assoc 2003;103:1191-4.

85 Malseed C, Nelson A, Ware R. Evaluation of a school-based health education program for urban Indigenous young people in Australia. Health 2014;06:587-97.

86 Pettigrew S, Jongenelis MI, Moore S, et al. A comparison of the effectiveness of an adult nutrition education program for Aboriginal and non-Aboriginal Australians. Soc Sci Med 2015;145:120-4.

87 Ronsley R, Lee AS, Kuzeljevic B, et al. Healthy Buddies ${ }^{\mathrm{TM}}$ reduces body mass index z-score and waist circumference in Aboriginal children living in remote coastal communities. J Sch Health 2013:83:605-13.

88 Santos RG, Durksen A, Rabbanni R, et al. Effectiveness of peerbased healthy living lesson plans on anthropometric measures and physical activity in elementary school students: a cluster randomized trial. JAMA Pediatr 2014;168:330-7.

89 Tomlin D, Naylor PJ, McKay H, et al. The impact of Action Schools $\mathrm{bc}$ on the health of Aboriginal children and youth living in rural and remote communities in British Columbia. Int $J$ Circumpolar Health 2012;71:17999-.
90 Bersamin A, Izumi BT, Nu J, et al. Strengthening adolescents' connection to their traditional food system improves diet quality in remote Alaska Native communities: results from the Neqa Elicarvigmun Pilot Study. Trans/ Behav Med 2019;9:952-61.

91 Brimblecombe J, Bailie R, van den Boogaard C, et al. Feasibility of a novel participatory multi-sector continuous improvement approach to enhance food security in remote Indigenous Australian communities. SSM Popul Health 2017;3:566-76.

92 Butler R, Tapsell L, Lyons-Wall P. Trends in purchasing patterns of sugar-sweetened water-based beverages in a remote Aboriginal community store following the implementation of a community-developed store nutrition policy. Nutrition Dietetics 2011;68:115-9.

93 Cueva K, Lovato V, Nieto T, et al. Increasing healthy food availability, purchasing, and consumption: lessons learned from implementing a mobile grocery. Progress in Community Health Partnerships 2018;12:65-72.

94 Fehring E, Ferguson M, Brown C, et al. Supporting healthy drink choices in remote Aboriginal and Torres Strait Islander communities: a community-led supportive environment approach Aust N Z J Public Health 2019;43:551-7.

95 Tyrrell M, Grundy J, Lynch P, et al. Laramba diabetes project: an evaluation of a participatory project in a remote Northern Territory community. Health Promot J Aust 2003;14:48-53.

96 Jones A, Magnusson R, Swinburn B, et al. Designing a healthy food partnership: lessons from the Australian food and health dialogue. BMC Public Health 2016;16:651.

97 Elliott T, Trevena H, Sacks G, et al. A systematic interim assessment of the Australian government's food and health dialogue. Med $J$ Aust 2014;200:92-5.

98 Jones A, Thow AM, Ni Mhurchu C, et al. The performance and potential of the Australasian Health StarRating system: a four-year review using the RE-AIM framework. Aust N Z J Public Health 2019;380

99 Lawrence MA, Dickie S, Woods JL, et al. Do nutrient-based front-of-pack labelling schemes support or undermine food-based dietary guideline recommendations? lessons from the Australian Health Star Rating system. Nutrients 2018;10:32.

100 Wang D, Stewart D. The implementation and effectiveness of school-based nutrition promotion programmes using a healthpromoting schools approach: a systematic review. Public Health Nutr 2013;16:1082-100.

101 Williams AJ, Henley WE, Williams CA, et al. Systematic review and meta-analysis of the association between childhood overweight and obesity and primary school diet and physical activity policies. Int J Behav Nutr Phys Act 2013;10:101.

102 Mah CL, Luongo G, Hasdell R, et al. A systematic review of the effect of retail food environment interventions on diet and health with a focus on the enabling role of public policies. Curr Nutr Rep 2019;8:411-28.

103 Lal A, Mantilla-Herrera AM, Veerman L, et al. Modelled health benefits of a sugar-sweetened beverage tax across different socioeconomic groups in Australia: a cost-effectiveness and equity analysis. PLoS Med 2017:14:e1002326.

104 Backholer K, Sarink D, Beauchamp A, et al. The impact of a tax on sugar-sweetened beverages according to socio-economic position: a systematic review of the evidence. Public Health Nutr 2016;19:3070-84

105 Black AP, Brimblecombe J, Eyles $\mathrm{H}$, et al. Food subsidy programs and the health and nutritional status of disadvantaged families in high income countries: a systematic review. BMC Public Health 2012;12:1099.

106 Thow AM, Downs S, Jan S. A systematic review of the effectiveness of food taxes and subsidies to improve diets: understanding the recent evidence. Nutr Rev 2014;72:551-65.

107 Colchero MA, Molina M, Guerrero-López CM. After Mexico implemented a tax, purchases of sugar-sweetened beverages decreased and water increased: difference by place of residence, household composition, and income level. J Nutr 2017;147:1552-7.

108 Colchero MA, Rivera-Dommarco J, Popkin BM, et al. In Mexico, evidence of sustained consumer response two years after implementing a sugar-sweetened beverage tax. Health Aff 2017;36:564-71.

109 Ferguson M, O'Dea K, Altman J, et al. Health-promoting food pricing policies and decision-making in very remote Aboriginal and Torres Strait Islander community stores in Australia. Int J Environ Res Public Health 2018;15:2908.

110 Navajo Nation Council. Healthy Dine nation act Navajo nation, 2014. Available: http://www.navajo-nsn.gov/News\%20Releases/ OPVP/2014/nov/Healthy\%20Dine\%20Nation\%20Act\%20of\% 202014.pdf 
111 Gregori D, Ballali S, Vecchio MG, et al. Randomized controlled trials evaluating effect of television advertising on food intake in children: why such a sensitive topic is lacking top-level evidence? Ecol Food Nutr 2014;53:562-77.

112 Hyseni L, Atkinson M, Bromley $\mathrm{H}$, et al. The effects of policy actions to improve population dietary patterns and prevent dietrelated non-communicable diseases: Scoping review. Eur J Clin Nutr 2017;71:694.

113 Brown V, Ananthapavan J, Veerman L, et al. The potential costeffectiveness and equity impacts of restricting television advertising of unhealthy food and beverages to Australian children. Nutrients 2018;10:622.

114 Ananthapavan J, Sacks G, Brown V, et al. Assessing costeffectiveness of obesity prevention policies in Australia 2018 (ACEObesity policy) 2018

115 Signal LN, Stanley J, Smith M, et al. Children's everyday exposure to food marketing: an objective analysis using wearable cameras. Int J Behav Nutr Phys Act 2017:14:137.

116 Smith M, Chambers T, Abbott M, et al. High stakes: Children's exposure to gambling and gambling marketing using wearable cameras. Int J Ment Health Addict 2019;34:1-23.

117 Chambers T, Pearson AL, Kawachi I, et al. Children's home and school neighbourhood exposure to alcohol marketing: using wearable camera and GPs data to directly examine the link between retailer availability and visual exposure to marketing. Health Place 2018;54:102-9.

118 Baum F, Fisher M. Why behavioural health promotion endures despite its failure to reduce health inequities. Sociol Health IIIn 2014;36:213-25.

119 Browne J, Adams K, Atkinson P, et al. Food and nutrition programs for Aboriginal and Torres Strait Islander Australians: an overview of systematic reviews. Aust Health Rev 2018;42:689-97.
120 Gwynn J, Sim K, Searle T, et al. Effect of nutrition interventions on diet-related and health outcomes of Aboriginal and Torres Strait Islander Australians: a systematic review. BMJ Open 2019;9:e025291. e.

121 Schembri L, Curran J, Collins L, et al. The effect of nutrition education on nutrition-related health outcomes of Aboriginal and Torres Strait Islander people: a systematic review. Aust N Z J Public Health 2016;40 Suppl 1:S42-7.

122 Jackson N, Waters E, Guidelines for Systematic Reviews in Health Promotion and Public Health Taskforce. Criteria for the systematic review of health promotion and public health interventions. Health Promot Int 2005;20:367-74.

123 MacLean S, Ritte R, Thorpe A, et al. Assessing compliance with Aboriginal and Torres Strait Islander health research guidelines within systematic reviews. Australian Indigenous Health Bulletin 2015;15:1-5.

124 National Health and Medical Research Council. Ethical conduct in research with Aboriginal and Torres Strait Islander peoples and communities: guidelines for researchers and stakeholders. Canberra: National Health and Medical Research Council, 2018.

125 Jamieson LM, Paradies YC, Eades S, et al. Ten principles relevant to health research among Indigenous Australian populations. Med $\mathrm{J}$ Aust 2012;197:16-18.

126 Morton Ninomiya ME, Atkinson D, Brascoupé S, et al. Effective knowledge translation approaches and practices in Indigenous health research: a systematic review protocol. Syst Rev 2017;6:28-38.

127 Pérez-Escamilla R, Lutter CK, Rabadan-Diehl C, et al. Prevention of childhood obesity and food policies in Latin America: from research to practice. Obes Rev 2017;18 Suppl 2:28-38. 